\title{
Avaliação da Casca e da Polpa Desidratada de Café (Coffea arabica L.) pela Técnica de Degradabilidade In vitro de Produção de Gás ${ }^{1}$
}

\section{Adauto Ferreira Barcelos ${ }^{2}$, Paulo César de Aguiar Paiva ${ }^{3}$, Juan Ramón Olalquiaga Peréz Júlio César Teixeira ${ }^{3}$, Roberto Maciel Cardoso ${ }^{4}$}

RESUMO - Com o objetivo de avaliar a casca e a polpa desidratada de café, quanto à degradabilidade in vitro pela técnica de produção de gás, conduziu-se o experimento, utilizando as cultivares de café Catuaí, Rubi e Mundo Novo. A polpa foi obtida pela despolpa úmida em despolpador mecânico e, em seguida, seca ao sol até alcançar 13\% de umidade. Os materiais foram armazenados em sacos de ráfia em ambiente coberto, ventilado e seco, por um ano, amostradas em triplicata a cada 90 dias. Incubaram-se in vitro $400 \mathrm{mg}$ de cada amostra (MS e FDN), em triplicata em banho maria a $39^{\circ} \mathrm{C}$. A produção cumulativa de gás foi obtida nos tempos $1,2,3,4,5,6,12,18,24$, $30,36,48,60$ e 72 horas. A cinética da produção cumulativa de gás para a MS e FDN foi analisada utilizando-se o modelo $\mathrm{V}_{\mathrm{t}}=\mathrm{V}_{\mathrm{t} 1} /$ $(1+\exp (2+4 \mathrm{~m}(\mathrm{~L}-\mathrm{T})))$ e SDN pelo modelo $\mathrm{V}_{\mathrm{t}}=\mathrm{V}_{\mathrm{t} 1} \times(1-\exp (-\mathrm{m} \times \mathrm{T}))$. A produção cumulativa de gás da fração SDN foi obtida pela diferença entre a produção cumulativa da MS e FDN. O armazenamento da casca e polpa desidratada de café melhorou a taxa de degradação e reduziu a fração fibrosa e não degradável, disponibilizando açúcares solúveis para a flora ruminal. Na casca de café de todas as cultivares, a maximização da contribuição da fração de SDN e FDN na fermentação ocorreu, respectivamente, em torno de 24 e 48 horas. A máxima produção de gás na MS da polpa ocorreu entre 48 e 60 horas, para todas as cultivares, e foi conseqüência da máxima produção de gás da fração FDN ocorrida em torno de 60 horas. Longo período de colonização pode constituir limitação no uso da casca e polpa desidratada de café, na alimentação de ruminantes, comprometendo a utilização do alimento pelos microorganismos do rúmen, devido à rápida passagem pelo rúmen.

Palavras-chave: cinética ruminal, degradabilidade in vitro, resíduo do café

\section{Evaluation of Coffee Grain (Coffea arabica L.) Hulls and Dehydrated Pulp In vitro Degradability Using the Gas Production Technique}

\footnotetext{
ABSTRACT - The experiment was conducted utilizing hull and dehydrated pulp of coffee cultivars Catuaí, Rubi and Mundo Novo. Pulp was obtained by moist pulping in a mechanical pulper and dried up to $13 \%$ moisture. Materials were stored in raffia bags in ventilated, moistureless environment and sun sampled every 90 days. $400 \mathrm{mg}$ of each sample (DM and NDF) were incubated in vitro in triplicates in a water bath at $39^{\circ} \mathrm{C}$. The cumulative gas production was obtained at 1,2,3,4,5,5,12, 18, 24, 30, 364860 and 72 hours. The kinetics of the cumulative gas output for DM and NDF as determined using the model $\mathrm{V}_{\mathrm{t}}=\mathrm{V}_{\mathrm{t} 1} /(1+\exp (2+4 \mathrm{~m}(\mathrm{~L}-\mathrm{T})))$ and SDN by the model $\mathrm{V}_{\mathrm{t}}=\mathrm{V}_{\mathrm{t} 1} \times(1-\exp (-\mathrm{m} \times \mathrm{T}))$. Cumulative gas production of the SDN fraction was obtained by the difference between the cumulative production from DM and NDF. Storage of hull and dehydrated coffee pulp improved the degradation rate, reduced fibrous and undegradable fractions, releasing soluble sugars for the ruminal flora. For coffee hulls of all cultivars, the maximum fermentation contribution of SDN and NDF took place respectively around 24 and 48 hours. The maximum gas production in pulp DM occurred between 48 and 60 hours in all cultivars and was a consequence of the maximum gas production by the NDF fraction, which peaked around 60 hours. Long colonization period may be a limitation to the use of hull and dehydrated coffee pulp in ruminant feeding, impairing feed utilization by ruminal microorganisms due to the rapid passage by the rumen.

Key Words: ruminal kinetics, in vitro degradability, coffee residue

\section{Introdução}

A utilização da casca de café na alimentação de ruminantes vem sendo estudada há algum tempo. Trabalhos desenvolvidos para avaliaro desempenho de bovinos de corte e leite, recebendo casca de café (BARCELOS

et al., 1997a,b), têm mostrado que sua utilização é economicamente viável para o produtor que tem disponibilidade do resíduo. O uso da casca de café na dieta de bovinos tem se intensificado nos últimos anos, em Minas Gerais e outros estados produtores de café, principalmente no período seco do ano.

${ }^{1}$ Parte da tese apresentada à Universidade Federal de Lavras - UFLA, pelo primeiro autor, para obtenção do título de Doutor em Zootecnia, na área de concentração Nutrição de Ruminantes. Projeto financiado pela FAPEMIG

${ }_{2}^{2}$ Pesquisador da EPAMIG/CTSM - CP 176 - 37200-000 - Lavras, MG. E.mail: adauto.barcelos@epamig.ufla.br

${ }^{3}$ Professor titular do Departamento de Zootecnia da UFLA, bolsista do CNPq.

${ }^{4}$ Professor aposentado da Universidade Federal de Viçosa
} 
Este interesse pela casca de café estimulou o desenvolvimento de trabalhos para se obter informações mais detalhadas sobre este mateiral (VILELA, 1999; SOUZA, 1999; TEIXEIRA, 1999; RIBEIRO FILHO, 1998; BARCELOS et al., 1997a; BARCELOS at al., 1997b; TEIXEIRA, 1995; FURUSHO, 1995). Entretanto, não foram realizados estudos do valor nutricional da casca de café, considerando alguns parâmetros como: cultivares, armazenamento, degradabilidade in vitro pela produção de gases, entre outros.

Outro material de interesse na alimentação de ruminantes é a polpa de café, a qual foi estudada em países como Colômbia e El Salvador, devido principalmente ao tipo de beneficiamento do café. Nestes, a tradição é despolpar o café e o resíduo é seco ao sol ou fermentado. Este tipo de tratamento melhora a qualidade do café, permitindo que se alcancem preços mais elevados no mercado internacional.

Visando melhorar a qualidade do café brasileiro, as instituições de pesquisa e fomento recomendam despolpar o café, o que tem resultado grande produção de polpa nas regiões produtoras.

A polpa úmida do café cereja é composta de epicarpo e parte do mesocarpo, material que sai do despolpador com alto teor de umidade. Sua utilização para alimentação de bovinos deve ser imediata, uma vez que sofre rápida fermentação. Uma alternativa para otimizar o seu uso seria a desidratação ao sol.

A casca e polpa de café têm sido estudadas no Brasil e em países da América Latina, contudo, não há relatos de estudos da cinética da digestão ruminal, empregando a técnica de produção de gás, visando sua recomendação para alimentação de ruminantes.

Muitos métodos estimam a digestibilidade ou degradabilidade para predizer o valor nutritivo dos alimentos. Técnicas in vitro permitem a avaliação rotineira da fermentação no rúmen empregando a fração líquida do conteúdo ruminal como inóculo, como a desenvolvida por TILLEY e TERRY (1963) ou do método de produção de gás (MENKE et al., 1979). Atualmente, metodologias de avaliação dos alimentos para ruminantes objetivam rapidez, baixo custo e confiabilidade. Uma técnica in vitro rápida segura e de boa precisão foi desenvolvida por PELL, SCHOFIELD e STONE (1994), a qual avalia a digestibilidade do alimento in vitro, em função da produção cumulativa dos gases $\mathrm{CO}_{2} \mathrm{e} \mathrm{CH}_{4}$.

Os métodos para avaliar alimentos para ruminantes, até início da década de 1980, forneciam apenas uma estimativa da digestibilidade potencial dos alimentos, com pouca referência à dinâmica da fermentação ruminal (VALENTIN et al., 1999). Uma segunda geração dos métodos foi desenvolvida, incorporando estimativas da cinética de degradação do retículo-rúmen. Entre os métodos para avaliação de alimentos, destaca-se o da produção de gás, liberado durante a fermentação de amostra incubada em líquido ruminal tamponado (THIAGO et al., 1998).

O método de MENKE et al. (1979) consiste na medida direta do volume de gás sob condições normais de pressão atmosférica, necessitando de constante assistência do técnico de laboratório. No entanto, VALENTIN et al. (1999), comparando as técnicas de degradabilidade in vitro e produção de gás pelo método de MENKE et al. (1979), concluíram que as correlações entre os resultados das duas metodologias não foram suficientemente fortes para predizer parâmetros de degradabilidade, provenientes dos parâmetros de produção de gás. Por outro lado, PELL e SCHOFIELD (1993) e THEODOROU et al. (1994) aperfeiçoaram a metodologia de produção de gás, o qual é acumulado em frascos de volume fixo conhecido e calculado segundo as variações na pressão, o que a tornou mais precisa, confiável e segura.

Considerando as vantagens da técnica de produção de gás, como sua simplicidade de uso e a possibilidade de processar grande número de amostras em curto espaço de tempo, é importante testar esta técnica com fontes alternativas de alimentos para ruminantes, buscando validar correlações entre a degradabilidade in situ e os parâmetros de produção de gás.

A técnica com monitoramento computadorizado de produção de gás para avaliar in vitro a digestão de forragens, desenvolvida por PELL e SCHOFIELD (1993), baseia-se no fato de os produtos da fermentação resultarem da fermentação dos carboidratos solúveis e insolúveis, portanto as taxas de digestão, calculadas utilizando as medidas de produção de gás, refletem as taxas das frações solúveis e insolúveis.

Segundo PELL e SCHOFIELD (1993), na condição em que nutrientes não sejam limitantes, a produção de gás é uma medida direta do crescimento microbiano e, em alguns aspectos, é o melhor índice para medir a energia metabolizável produzida.

A técnica de produção de gás considera a conversão de todas as principais fontes ricas em energia metabolizavel como monossacarídeos, polissacarídeos, pectinas, amido, celulose e hemicelulose, em $\mathrm{CO}_{2} \mathrm{e}$ $\mathrm{CH}_{4}$. Assim, esse método pode ser utilizado para 
determinar a importância de algumas dessas diferentes frações do alimento, em fornecer energia aos microorganismos (PELL e SCHOFIELD, 1993).

O objetivo deste trabalho foi estimar os parâmetros cinéticos da degradação da matéria seca (MS) e fibra insolúvel em detergente neutro $(\mathrm{FDN})$ e da fração solúvel em detergente neutro (SDN) da casca e da polpa desidratada de café, obtidas de três cultivares e armazenados por 12 meses, utilizando-se a técnica in vitro de produção cumulativa de gás.

\section{Material e Métodos}

O experimento foi conduzido no Laboratório de Nutrição Animal do Departamento de Zootecnia da Universidade Federal de Lavras e Laboratório de Qualidade do Café Dr. Alcides de Carvalho do CTSM, da EPAMIG, no município de Lavras, no período de outubro de 1997 a dezembro de 1998.

Utilizou-se casca e polpa desidratada de três cultivares de café (Catuaí vermelho IAC2077-2-599, Rubi MG1192 e Mundo Novo IAC379-19), provenientes da Fazenda Experimental de São Sebastião do Paraíso, da EPAMIG.

A casca foi obtida da limpeza do café em coco por cultivar, e a polpa foi obtida também de cada cultivar, pela despolpa úmida, utilizando-se de despolpador DC3 e degomado no degomador mecânico DM2, ambos Pinhalense ${ }^{\circledR}$ (Pinhal - SP) e, em seguida, seca ao sol até $13 \%$ de umidade.

A casca e a polpa foram armazenadas em sacos de ráfia, em ambiente coberto, ventilado e seco, por um ano. Nesse período coletou-se amostra em triplicata a cada 90 dias para análises bromatológicas e das frações A, $B_{1}, B_{2}$ e C de carboidratos, segundo SNIFFEN et al. (1992).

As amostras foram pré-secas em estufa a $65^{\circ} \mathrm{C}$ com ventilação, até alcançar peso constante, o que ocorreu entre 36 e 48 horas, e moídas em peneira de $2 \mathrm{~mm}$.

Foram incubados in vitro $400 \mathrm{mg}$ de cada amostra correspondente a MS e peso equivalente em FDN em triplicata, em banho maria a $39^{\circ} \mathrm{C}$, conforme descrito em MALAFAIA (1997), As leituras de pressão dos gases foram obtidas por um manômetro $\left(0-1 \mathrm{kgf} / \mathrm{cm}^{2}\right)$, acoplado a seringa de $20 \mathrm{~mL}$, conforme descrito em MALAFAIA (1997), nos tempos 1, 2, $3,4,5,6,12,18,24,30,36,48,60$ e 72 horas. A cinética da produção cumulativa de gás para a MS e FDN foi analisada utilizando-se o modelo de SCHOFIELD, PITT e PELL (1994):

$$
V_{t}=\frac{V_{t 1}}{\left(1+e^{(2+4 \mu(T-L))}\right)}
$$

em que: $\mathrm{V}_{\mathrm{t}}=$ volume de gás do substrato produzido no tempo $\mathrm{T} ; \mathrm{V}_{\mathrm{t} 1}=$ volume de gás da digestão completa do substrato; $\mu=$ taxa de degradação do substrato; $\mathrm{L}=$ tempo de colonização e $\mathrm{T}=$ tempo de incubação do substrato, e a fração solúvel em detergente neutro (SDN) pelo modelo:

$$
V_{t}=V_{t 1} \times\left(1-e^{(-\mu \times T)}\right)
$$

A produção cumulativa de gás da fração SDN foi obtida pela diferença entre a produção cumulativa da MS e FDN. Os coeficientes m (taxa de degradação) e L (tempo de colonização) foram obtidos utilizandose o programa Sistema de Análises Estatísticas SAEG, versão 5.0.

O delineamento experimental utilizado foi o inteiramente casualizado, com três repetições, em esquema fatorial $3 \times 2 \times 5$ (cultivar $\mathrm{x}$ material $\mathrm{x}$ armazenamento). Os materiais foram a casca e polpa desidratada e o armazenamento de 0, 90, 180, 270 e 360 dias, conforme modelo estatístico:

$\mathrm{Y}_{\mathrm{ijkl}}=\mu+\mathrm{C}_{\mathrm{i}}+\mathrm{M}_{\mathrm{j}}+\mathrm{P}_{\mathrm{k}}+\mathrm{CM}_{\mathrm{ij}}+\mathrm{MP}_{\mathrm{jk}}+\mathrm{CMP}_{\mathrm{ijk}}+\mathrm{e}_{\mathrm{ijkl}}$ em que: $\mathrm{Y}_{\mathrm{ijkl}}=$ observação referente a cultivar $i$ do material $j$ no tempo $k$ e repetição $l ; \mu=$ média geral; $\mathrm{C}_{\mathrm{i}}=$ efeito da variedade, sendo $i=1$ a 3 ; $\mathrm{M}_{\mathrm{j}}=$ efeito de material, sendo $j=1$ a $2 ; \mathrm{P}_{\mathrm{k}}=$ efeito do período de armazenamento, sendo $k=1$ a 5 ; $\mathrm{CM}_{\mathrm{ij}}=$ efeito da interação de cultivar $i$ e material $j$; $\mathrm{CP}_{\mathrm{jk}}=$ efeito da interação cultivar $i$ e período de armazenamento $k ; \mathrm{MP}_{\mathrm{jk}}=$ efeito da interação material $j$ e período de armazenamento $k$; $\mathrm{CMP}_{\mathrm{ijk}}=$ efeito da interação cultivar $i$, material $j$ e período de armazenamento ${ }_{\mathrm{k}} ; \mathrm{e}_{\mathrm{ijkl}}=$ erro aleatório associado a cada observação, sendo $l=1$ a 3 .

Os dados foram analisados utilizando-se programa estatístico Sistema de Análise de Variância para Dados Balanceados - Sisvar, segundo FERREIRA (2000), e as médias comparadas pelo teste de SCOTT e KNOTT (1974).

Neste trabalho, foram analisadas as variáveis volume cumulativo final de gás (VF), taxa de degradação (m) e L (tempo de colonização), para a matéria seca (MS); fibra insolúvel em detergente neutro (FDN) e VF e m para a fração solúvel em detergente neutro (SDN), da casca e polpa desidratada das cultivares de café Catuaí, Rubi e Mundo Novo, armazenadas durante 12 meses. 


\section{Resultados e Discussão}

Houve efeito significativo de cultivar $(\mathrm{P} \leq 0,03)$, material $(\mathrm{P} \leq 0,01)$ e interação de ambos $(\mathrm{P} \leq 0,01)$, quanto à taxa de degradação (Tabela 1). Quando se comparou casca e polpa em relação a tempo de colonização (L), observou-se (Tabela 1, Figuras 2 e 3 ) que o tempo de colonização para a polpa foi maior $(17,83 \mathrm{~h})$ em relação à casca $(3,52 \mathrm{~h})$. O tempo de colonização não variou entre cultivares nos dois materiais.

O alto valor de L na polpa, em comparação à casca, provavelmente é decorrente da sua composição em carboidratos, a qual possui maior quantidade de carboidratos estruturais $\left(\mathrm{B}_{1}, \mathrm{~B}_{2}\right.$ e $\left.\mathrm{C}\right)$ e menor de carboidrato não estrutural (A), comparada à casca de café (Tabelas 2 e 3), embora não possa ser descartada a possibilidade da polpa conter compostos secundários inibidores da atividade microbiana. Este valor $(17,83 \mathrm{~h})$ é próximo ao valor de L da FDN da casca e da polpa, apresentados na Tabela 4, indicando que há grande quantidade de material potencialmente fermentável em relação ao solúvel.

Na obtenção da polpa de café, há perda de boa parte dos carboidratos solúveis removidos pela água utilizada na despolpa. A casca obtida pelo beneficiamento do grão de café em coco possui todas as frações, inclusive os açúcares solúveis. Esta diferença pode explicar o menor tempo de colonização para a casca comparada à polpa, isto é, L é menor para a casca, devido à fermentação da fração solúvel de degradação rápida.

A MS na casca das cultivares Catuaí e Mundo Novo tiveram maior taxa de degradação em relação à cultivar Rubi, enquanto na polpa a maior taxa de degradação foi encontrada na cultivar Rubi (Tabela 1). Não foram encontradas, na literatura, explicações para a diferença entre as cultivares, no entanto, a cultivar Rubi foi obtida pelo cruzamento entre as cultivares Mundo Novo e Catuaí, com retrocruzamento para a cultivar Mundo Novo. Como a taxa de degradação da MS da polpa da cultivar Mundo Novo foi superior à da cultivar Catuaí, supostamente esta característica foi transmitida para a cultivar Rubi.

A taxa de degradação da MS aumentou com o tempo de armazenamento. A Figura 1 mostra o efeito do tempo de armazenamento sobre a taxa de degradação da MS. Para cada dia de armazenamento da casca e polpa houve

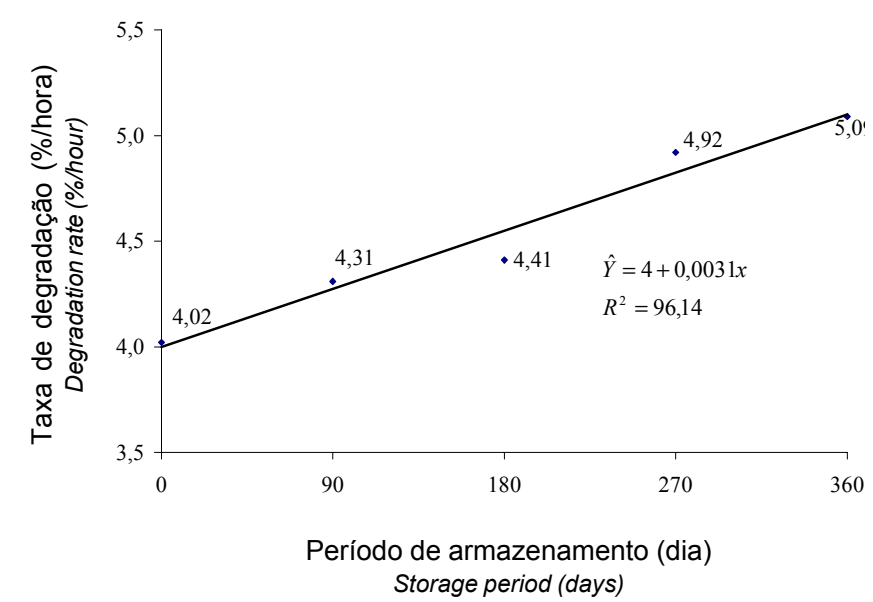

Figura 1 - Efeito do período de armazenamento sobre a taxa de degradação da MS (\% por hora) da casca e polpa de café.

Figure 1 - Effect of the storage period on DM degradation rate $(\%$ per hour) of the coffee hull and pulp.

aumento de $0,0031 \%$, na taxa de degradação, o que representou aumento total de $27 \%$.

BARCELOS (2000) encontrou acréscimo no teor dos açúcares, à medida que aumentou o tempo de armazenamento (fração A), sendo esse acréscimo de $14,7 \%$ em 360 dias de armazenamento. $\mathrm{O}$ aumento no teor de açúcares em decorrência do tempo de armazenamento é devido, possivelmente, à degradação dos tecidos vegetais por microrganismos saprófitos aeróbicos, principalmente das frações $\mathrm{B}_{2}$ e $\mathrm{C}$, uma vez que essas frações reduziram-se com o tempo de armazenamento (BARCELOS, 2000).

O armazenamento da casca e polpa desidratada de café, por um período de doze meses, pode melhorar a taxa de degradação por reduzir a fração fibrosa e não degradável e disponibilizar açúcares prontamente solúveis, para os microorganismos do rúmen (BARCELOS, 2000).

Foram encontrados efeitos significativos na taxa de degradação e tempo de colonização da FDN $(\mathrm{P} \leq 0,01 ; \mathrm{P} \leq 0,00$ e $\mathrm{P} \leq 0,00)$ e $\mathrm{SDN}(\mathrm{P} \leq 0,03$; $\mathrm{P} \leq 0,00$ e $\mathrm{P} \leq 0,00)$, respectivamente, de cultivar, material e interação de ambos. A casca de café apresentou maior taxa de degradação e menor tempo de colonização que a polpa desidratada (Tabela 4) para a FDN, correspondendo, respectivamente, a 14,7 e $5,2 \%$. Os valores do tempo de colonização da FDN (Tabela 4) e MS (Tabela 1) da polpa desidratada são bastante próximos, indican- 
Tabela 1 - Valores da taxa de degradação $(\mu)$ e tempo de colonização (L) da MS da casca e polpa desidratada de café, segundo as cultivares

Table 1 - Cultivars values for degradation rate $(\mu)$ and colonization time $(L)$ of coffee hull and dehydrated pulp, DM

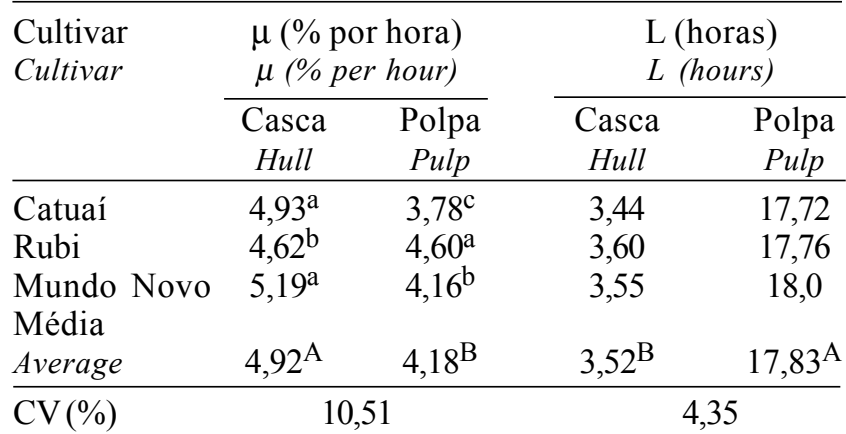

Médias nas colunas seguidas de letras minúsculas diferentes diferem pelo teste de SKOTT e KNOTT a $5 \%$ de probabilidade

Medias nas linhas seguidas de letras maiúsculas diferentes diferem pelo teste $\mathrm{t}$ a $5 \%$ de probabilidade.

Averages in the columns followed by different small letters differ by SKOTT and KNOTT test at $5 \%$ of probability.

Averages in the lines followed by different capital letters differ by $t$ test at $5 \%$ of probability.

do que esse material, comparado à casca de café, possui maior proporção de carboidratos de degradação lenta (frações B e C).

Tal fato foi relatado por Barcelos (2000), o qual encontrou valor significativamente maior $(67,4 \%)$ para polpa desidratada comparada à casca $(65,7 \%)$ para a fração de degradação lenta $\left(\mathrm{B}_{2}\right)$ dos carboidratos.

A fração solúvel em detergente neutro (SDN) da casca de café, comparada à polpa, apresentou maior $(\mathrm{P} \geq 0,00)$ taxa de degradação (Tabela 4) decorrente da maior proporção de frações A e $\mathrm{B}_{1}$ na casca (Tabela 2), principalmente a de degradação rápida $(\mathrm{A})$.

Devido à ausência de trabalhos sobre a produção de gás com a casca e polpa desidratada de café, os resultados deste estudo foram comparados aos de CABRAL et al. (1997) e MALAFAIA et al. (1997), trabalhando com outros materiais fibrosos.

Os valores da taxa de degradação da MS, FDN e SDN, da casca e polpa desidratada de café, estão próximos aos encontrados por CABRAL et al. (1997) no capim-elefante cortado na primavera e verão, aos 42 e 63 dias de rebrota e por MALAFAIA, VALADARES FILHO e VIERIA (1997) obtidos com capim Tifton-85, elefante e silagem de milho, em modelo de duplo compartimento.

A fração solúvel da casca de café contribuiu para o aumento total de gás (Figura 2) produzido nas três cultivares estudadas. Segundo MALAFAIA et al. (1997), tal fato pode ser relacionado à presença de carboidratos não estruturais, rapidamente fermentáveis. Isso é mostrado por BARCELOS (2000), que encontrou menor valor da fração A na polpa desidratada de café $(7,2 \%)$ comparada à casca de café $(10,1 \%)$, ou seja, a casca de café possui $40,3 \%$ mais carboidratos de degradação rápida, quando comparada à polpa.

$\mathrm{Na}$ casca de café de todas as cultivares, a maximização da contribuição da SDN na fermentação, representada pela produção cumulativa de gás, ocorreu em torno de 24 horas (Figura 2), ao passo que na MS e FDN a maximização da produção de gás ocorreu às 48 horas. Esses resultados mostram que, na casca de café, após 48 horas, independentemente de variedade, ocorre drástica queda na taxa de degradação.

Os valores da taxa de degradação da MS e FDN, da polpa desidratada de café, são semelhantes (Tabelas 1 e 4), mostrando que a fração solúvel da polpa desidratada é baixa e, conseqüentemente, o tempo de colonização foi maior (Tabela 1 e Figura 3 ).

Tabela 2 - Teores médios das frações dos carboidratos, na casca e polpa desidratada de café, de acordo com as cultivares (\% dos carboidratos totais)

Table 2 - Average contents of the carbohydrates fractions, in the hull and dehydrated pulp of coffee, according to the cultivars (\% of total carbohydrates)

\begin{tabular}{|c|c|c|c|c|c|c|c|c|}
\hline \multirow[t]{3}{*}{$\begin{array}{l}\text { Cultivares } \\
\text { Cultivars }\end{array}$} & \multicolumn{8}{|c|}{$\begin{array}{l}\text { Frações ( } \% \text { dos carboidratos totais) } \\
\text { Fractions (\% of total carbohydrate) }\end{array}$} \\
\hline & \multicolumn{2}{|c|}{ A } & \multicolumn{2}{|c|}{$\mathrm{B}_{1}$} & \multicolumn{2}{|c|}{$\mathrm{B}_{2}$} & \multicolumn{2}{|c|}{$\mathrm{C}$} \\
\hline & $\begin{array}{c}\text { Casca } \\
\text { Hull }\end{array}$ & $\begin{array}{l}\text { Polpa } \\
\text { Pulp }\end{array}$ & $\begin{array}{c}\text { Casca } \\
\text { Hull }\end{array}$ & $\begin{array}{l}\text { Polpa } \\
\text { Pulp }\end{array}$ & $\begin{array}{c}\text { Casca } \\
\text { Hull }\end{array}$ & $\begin{array}{l}\text { Polpa } \\
\text { Pulp }\end{array}$ & $\begin{array}{c}\text { Casca } \\
\text { Hull }\end{array}$ & $\begin{array}{l}\text { Polpa } \\
\text { Pulp }\end{array}$ \\
\hline Catuaí & 11,17 & 8,34 & 0,12 & 0,08 & 65,20 & 66,85 & 23,42 & 25,11 \\
\hline Rubi & 10,03 & 7,28 & 0,11 & 0,07 & 65,43 & 67,47 & 23,94 & 25,57 \\
\hline Mundo Novo & 9,11 & 6,07 & 0,09 & 0,06 & 66,74 & 68,00 & 24,13 & 25,60 \\
\hline $\begin{array}{l}\text { Média } \\
\text { Average }\end{array}$ & 10,10 & 7,23 & 0,11 & 0,07 & 65,79 & 67,44 & 23,83 & 25,43 \\
\hline
\end{tabular}


Tabela 3 - Teores médios dos carboidratos da casca e polpa desidratada de café, segundo as cultivares (\% na MS)

Table 3 - Mediumtenors of the carbohydrates of hull and dehydrated pulp of coffee, second the cultivars (\% in the DM)

\begin{tabular}{|c|c|c|c|c|c|c|}
\hline \multirow{3}{*}{$\begin{array}{l}\text { Cultivares } \\
\text { Cultivars }\end{array}$} & \multicolumn{6}{|c|}{$\begin{array}{l}\text { Carboidratos (\% na MS) } \\
\text { Carbohydrates (\% in DM) }\end{array}$} \\
\hline & \multicolumn{2}{|c|}{ Total } & \multicolumn{2}{|c|}{$\begin{array}{l}\mathrm{CNE} \\
N E C\end{array}$} & \multicolumn{2}{|c|}{$\begin{array}{l}\mathrm{CE} \\
E C\end{array}$} \\
\hline & $\begin{array}{c}\text { Casca } \\
\text { Hull }\end{array}$ & $\begin{array}{l}\text { Polpa } \\
\text { Pulp }\end{array}$ & $\begin{array}{c}\text { Casca } \\
\text { Hull }\end{array}$ & $\begin{array}{l}\text { Polpa } \\
\text { Pulp }\end{array}$ & $\begin{array}{c}\text { Casca } \\
\text { Hull }\end{array}$ & $\begin{array}{l}\text { Polpa } \\
\text { Pulp }\end{array}$ \\
\hline Catuaí & 79,26 & 76,36 & 12,79 & 8,81 & 66,46 & 67,54 \\
\hline Rubi & 79,27 & 76,23 & 13,01 & 7,79 & 66,26 & 68,43 \\
\hline $\begin{array}{l}\text { Mundo } \\
\text { Novo }\end{array}$ & 77,71 & 75,40 & 11,50 & 6,97 & 66,20 & 68,44 \\
\hline $\begin{array}{l}\text { Média } \\
\text { Average }\end{array}$ & 78,75 & 74,99 & 12,43 & 7,86 & 66,31 & 68,14 \\
\hline
\end{tabular}

$\mathrm{Na}$ polpa de café de todas as cultivares, a maximização da contribuição da FDN na fermentação, representada pela produção máxima de gás, ocorreu às 60 horas (Figura 3), superior ao da casca de café (Figura 2), indicando que até 60 horas existiam compostos a serem fermentados.

Não foram encontradas diferenças significativas $(\mathrm{P} \geq 0,01)$ na produção cumulativa de gás para cultivar, material e período de armazenamento e interações. $\mathrm{Na}$ Tabela 5 estão apresentados os valores de produção cumulativa de gás na MS, FDN e SDN por material segundo o tempo de armazenamento.

A produção máxima de gás na MS da polpa (60 horas) ocorreu acima do tempo da casca de café (48 horas), decorrente da contribuição da produção de gás da FDN e SDN, que foi máxima com 60 horas.

Longo período de colonização, como o ocorrido com a polpa comparada à casca de café (Tabela 1), pode comprometer a utilização do alimento pelos microorganismos do rúmen, devido à taxa de passagem. O alimento passa à parte distal do trato gastrointestinal durante o tempo de colonização $(17 \mathrm{~h})$ e digestão dos carboidratos estruturais e proteína ligada à parede celular. Isso pode ser uma limitação da polpa desidratada de café como alimento para ruminantes. Assim, a maior contribuição da casca está na SDN, que é baixa.

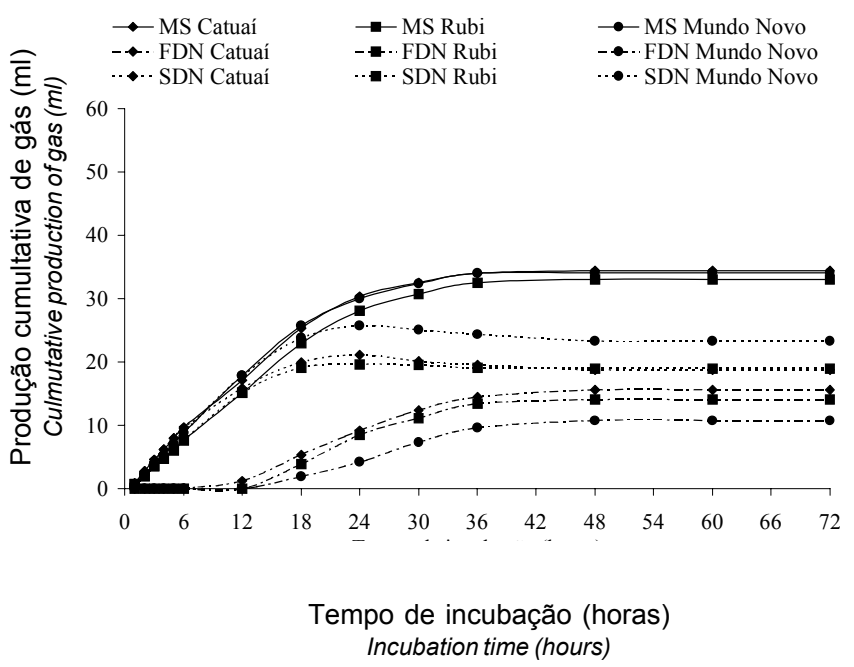

Figura 2 - Produção cumulativa de gás (ml) oriunda da matéria seca (MS), fibra insolúvel em detergente neutro (FDN) e fração solúvel em detergente neutro (SDN) da casca de café Catuaí, Rubi e Mundo Novo, em função do tempo de incubação.

Figure 2 - Cumulative production of gas $(\mathrm{ml})$ originating from of dry matter (DM), insoluble fiber in neutral detergent (NDF) and soluble fraction in neutral detergent (NDS) of the coffee hull Catuaí, Rubi and Mundo Novo, in function of the incubation time.

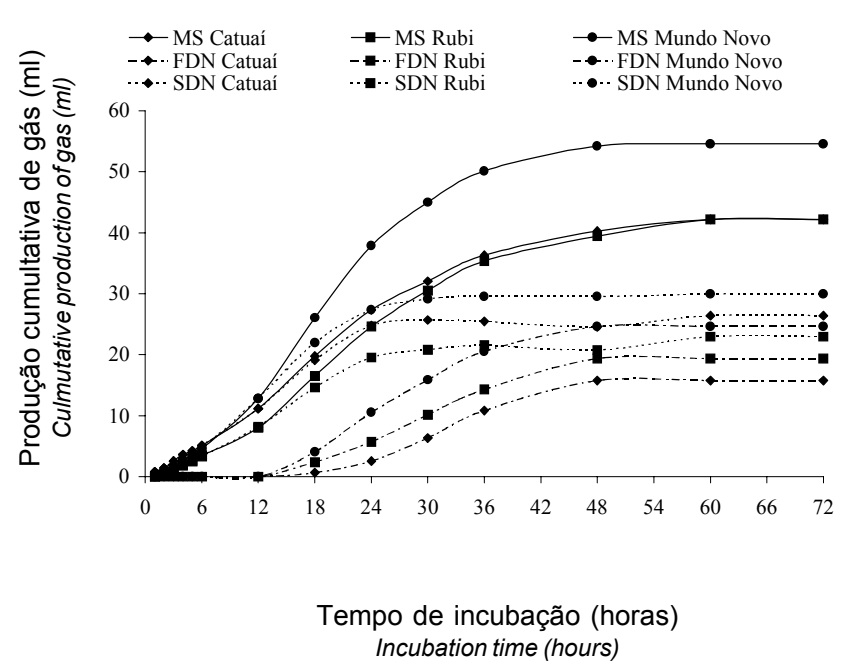

Figura 3 - Produção cumulativa de gás $(\mathrm{mL})$ oriunda da matéria seca (MS), fibra insolúvel em detergente neutro (FDN) e fração solúvel em detergente neutro (SDN) da polpa desidratada de café Catuaí, Rubi e Mundo Novo, em função do tempo de incubação.

Figure 3 - Cumulative production of gas $(\mathrm{ml})$ from dry matter (DM), insoluble fiber in neutral detergent (NDF) and soluble fraction in neutral detergent (NDS) of the dehydrate pulp Catuaí, Rubi and Mundo Novo, in function of incubation time. 
Tabela 4 - Valores médios da taxa de degradação ( $m$, em \% por hora) e tempo de colonização (L, em horas), da FDN e SDN da casca e polpa desidratada, segundo as cultivares

Table 4 - Average values of the degradation rate ( $m$, \% per hour) and time of colonization ( $L$, in hours), of NDF and NDS of the hull and dehydrated pulp, according to the cultivars

\begin{tabular}{|c|c|c|c|c|c|c|}
\hline \multirow{3}{*}{$\begin{array}{l}\text { Cultivar } \\
\text { Cultivar }\end{array}$} & \multicolumn{4}{|c|}{$\begin{array}{l}\text { FDN } \\
N D F\end{array}$} & \multirow{2}{*}{\multicolumn{2}{|c|}{$\begin{array}{c}\text { SDN } \\
N D S \\
\mu(\% \text { por hora }) \\
\mu(\% \text { per hour })\end{array}$}} \\
\hline & \multicolumn{2}{|c|}{$\begin{array}{l}\mu(\% \text { por hora }) \\
\mu(\% \text { per hour })\end{array}$} & \multicolumn{2}{|c|}{$\begin{array}{l}\mathrm{L} \text { (horas) } \\
L \text { (hours) }\end{array}$} & & \\
\hline & $\begin{array}{c}\text { Casca } \\
\text { Hull }\end{array}$ & $\begin{array}{l}\text { Polpa } \\
\text { Pulp }\end{array}$ & $\begin{array}{c}\text { Casca } \\
\text { Hull }\end{array}$ & $\begin{array}{l}\text { Polpa } \\
\text { Pulp }\end{array}$ & $\begin{array}{c}\text { Casca } \\
\text { Hull }\end{array}$ & $\begin{array}{l}\text { Polpa } \\
\text { Pulp }\end{array}$ \\
\hline $\begin{array}{l}\text { Catuaí } \\
\text { Rubi } \\
\text { Mundo Novo }\end{array}$ & $\begin{array}{l}5,69^{\mathrm{a}} \\
5,36^{\mathrm{b}} \\
6,02^{\mathrm{a}}\end{array}$ & $\begin{array}{l}4,38^{\mathrm{c}} \\
5,33^{\mathrm{a}} \\
4,83^{\mathrm{b}}\end{array}$ & $\begin{array}{l}13,30^{\mathrm{b}} \\
22,52^{\mathrm{a}} \\
15,34^{\mathrm{b}}\end{array}$ & $\begin{array}{l}18,63^{\mathrm{a}} \\
17,98^{\mathrm{a}} \\
16,32^{\mathrm{b}}\end{array}$ & $\begin{array}{l}7,83^{\mathrm{a}} \\
7,52^{\mathrm{b}} \\
8,08^{\mathrm{a}}\end{array}$ & $\begin{array}{l}6,67^{\mathrm{c}} \\
7,50^{\mathrm{a}} \\
7,06^{\mathrm{b}}\end{array}$ \\
\hline $\begin{array}{l}\text { Média } \\
\text { Average }\end{array}$ & $5,69^{\mathrm{A}}$ & $4,85^{\mathrm{B}}$ & $16,73^{\mathrm{B}}$ & $17,64^{\mathrm{A}}$ & $7,81^{\mathrm{A}}$ & $7,08^{\mathrm{B}}$ \\
\hline $\mathrm{CV}(\%)$ & & & & & & \\
\hline
\end{tabular}

Médias nas colunas seguidas de letras minúsculas diferentes diferem pelo teste de SKOTT e KNOTT a $5 \%$ probabilidade.

Médias nas linhas seguidas de letras maiúsculas diferentes diferem pelo teste $t$ a $5 \%$ probabilidade.

Averages in the columns followed by different small letters differ by SKOTT and KNOTT test, at $5 \%$ of probability.

Averages in the lines followed by different capital letters differ by $t$ test, at $5 \%$ of probability.

Tabela 5 - Produção cumulativa de gás da MS, FDN e SDN segundo os tempos de armazenagem da casca e polpa desidratada de café.

Table 5 - Cunulative production of gas of DM, NDF and NDS according to storage times of the hull and dehydrated coffee pulp

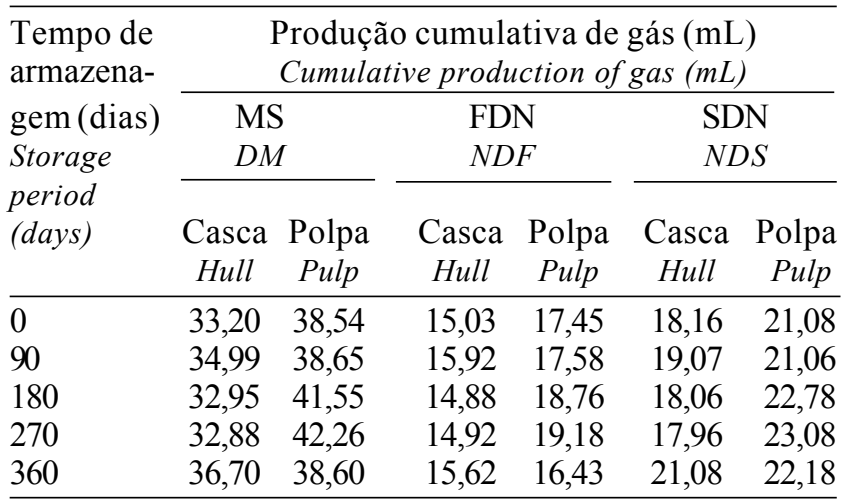

\section{Conclusões}

O armazenamento da casca e polpa desidratada de café por um período de doze meses melhorou a taxa de degradação da MS em $27 \%$.

A casca de café da cultivar Mundo Novo apresentou maior taxa de degradação da MS, FDN e SDN, ao passo que, na polpa, a maior taxa de degradação foi determinada na cultivar Rubi.

Longo tempo de colonização na polpa de café pode ser fator de limitação do seu uso na alimentação de ruminantes, decorrente da taxa de passagem.
Na casca de café, a maximização na produção de gás ocorrida às 36 horas foi decorrente da fermentação da SDN, ao passo que na polpa, ocorrida às 60 horas, foi devido à fermentação da FDN. Os resultados mostram que, após 36 horas na casca de café e 60 horas na polpa, independentemente de cultivar, as frações $\mathrm{A}, \mathrm{B}_{1}$ e $\mathrm{B}_{2}$ foram fermentadas.

\section{Referências Bibliográficas}

BARCELOS, A.F. Parâmetros bromatológicos, frações de carboidratos e degradabilidadede in vitro da casca e da polpa de café (Coffea arabica L.). Lavras: UFLA, 2000.96p. Tese (Doutorado em Zootecnia) - Universidade Federal de Lavras, 2000.

BARCELOS, A.F., ANDRADE, I.F., TIESENHAUSEN, I.M.E.V. Von et al. 1997a. Aproveitamento da casca de café na alimentação de novilhos confinados. I - Resultados do primeiro ano. R. Bras. Zootec., 26(6):1208-1214.

BARCELOS, A.F., ANDRADE, I.F., TIESENHAUSEN, I.M.E.V. Von et al. 1997b. Aproveitamento da casca de café na alimentação de novilhos confinados. I - Resultados do segundo ano. R. Bras. Zootec., 26(6):1215-1221.

CABRAL, L.S., MALAFAIA, P.A.M., VALADARES FILHO, S.C. et al. Cinética ruminal da FDN do capim elefante estimada pela técnica da produção de gás e pela degradação In vitro. In: REUNIÃO ANUAL DA SOCIEDADE BRASILEIRA DE ZOOTECNIA, 34, 1997, Juiz de Fora. Anais... Juiz de Fora: SBZ, 1997. p.91-93.

FURUSHO, I.F. Efeito da utilização da casca de café, in natura e tratada com uréia, sobre o desempenho e características de carcaça de cordeiros terminados em confinamento. Lavras: UFLA, 1995. 72p. Dissertação (Mestrado em Zootecnia) Universidade Federal de Lavras, 1995.

MALAFAIA, P.A.M., VALADARES FILHO, S.C., VIEIRA, R.A.M. Avaliação de alguns volumosos através da técnica de 
produção de gás e da subtração de curvas. In: REUNIÃO ANUAL DA SOCIEDADE BRASILEIRA DE ZOOTECNIA, 34, 1997, Juiz de Fora. Anais... Juiz de Fora: SBZ, 1997. p.103-105.

MENKE, K.H., RAAB, L., SALENWSKI, A. et al. 1979. The estimation of the digestibility and metabolizable energy content of ruminant feedstuffs from the gas production when they are incubated with rumen liquor in vitro. J. Agric. Sci., 93(1):217-223.

PELL, A.N., SCHOFIELD, P. 1983. Computerized monitoring of gas production to measure forage digestion in vitro. J. Dairy Sci., 6(4):1063-1073.

PELL. A.N., SCHOFIELD, P., STONE, W.C. Rates of digestion of feeds measured in vitro with computers. In: CORNELL NUTRITION CONFERENCE, 1994, Ithaca. Proceedings... Cornell, 1994. p.74-81.

RIBEIRO FILHO, E. Degradabilidade in situ da matéria seca, proteina bruta e da fibra em detergente neutro da casca de café e desempenho de novilhos mestiços em fase de recria. Lavras: UFLA, 1998. 55p. Dissertação (Mestrado em Zootecnia) - Universidade Federal de Lavras, 1998.

SCHOFIELD, P., PITT, R.E., PELL, A.N. 1994. Kinetics of fiber digestion from in vitro gas production. J. Anim. Sci., 72(11):2980-2991.

SOUZA, A.L. Composição químico-bromatológica da casca de café tratada com amônia anidra e sulfeto de sódio. Viçosa, MG: UFV, 1999. 58p. Dissertação (Mestrado em Zootecnia) - Universidade Federal de Viçosa, 1999.

TEIXEIRA, J.C. Café. In: SIMPÓSIO SOBRE NUTRIÇÃO DE BOVINOS, 6, 1995, Piracicaba. Anais... Piracicaba: FEALQ, 1995. p.123-151.

TEIXEIRA, M.N.M. Determinação da degradabilidade in situ das diferentes frações da casca de três cultivares de café (Coffea arabica L). Lavras: UFLA, 1999. 44 p. Dissertação (Mestrado em Zootecnia) - Universidade Federal de Lavras, 1999.
THEODOROU, M.K., WILLIAMS, B.A., DHANOA, M.S. et al. 1994. A simple gas production method using a pressure transducer to determine the fermentation kinetics of ruminant feeds. Anim. Feed Sci. Techn., 48:185-197.

THIAGO, L.R.L.S., COLUCCI, P., PLAIZIER, J.C. et al. Produção de gás: uma alternativa para digestibilidade in vitro. In: REUNIÃO ANUAL DA SOCIEDADE BRASILEIRA DE ZOOTECNIA, 34., 1997, Juiz de Fora. Anais... Juiz de Fora: SBZ, 1997. p.100-102.

TILLEY, J.M.A., TERRY, R.A. 1963. A two stage technique for the in vitro digestion of forage crops. J. Brit. Grassland Society, 18:104-111.

VALENTIN, S.F., WILLIAMS, P.E.V., FORBES, J.M.et al. 1999. Comparison of the in vitro gas production technique and the nylon bag degradability technique to measure shortan long-term processes of degradation of maize silage in dairy cows. Anim. Feed Sci. Techn., 78(1/2):81-99.

VILELA, F.G. Uso da casca de café melosa em diferentes niveis na alimentação de novilhos confinados. Lavras: UFLA, 1999. 46p. Dissertação (Mestrado em Zootecnia) - Universidade Federal de Lavras, 1999. 